\title{
Impact of free fatty acids on prognosis in coronary artery disease patients under different glucose metabolism status
}

\author{
Jing-Lu Jin ${ }^{\dagger}$, Ye-Xuan Cao ${ }^{\dagger}$, Hui-Hui Liu, Hui-Wen Zhang, Yuan-Lin Guo, Na-Qiong Wu, Cheng-Gang Zhu,
} Rui-Xia Xu, Ying Gao, Jing Sun, Qian Dong and Jian-Jun Li* (D)

\begin{abstract}
Background: The aim of the present study is to examine the effects of free fatty acids (FFAs) on major cardiovascular events (MACEs) in patients with stable coronary artery disease (CAD) and different glucose metabolism status.

Methods: In this study, we consecutively enrolled 5443 patients from March 2011 to May 2015. Patients were categorized according to both status of glucose metabolism status [diabetes mellitus (DM), pre-diabetes (Pre-DM), normal glycaemia regulation (NGR)] and FFAs levels. All subjects were followed up for the occurrence of the MACEs.

Results: During a median of 6.7 years' follow-up, 608 MACEs occurred. A twofold higher FFAs level was independently associated with MACEs after adjusting for confounding factors [Hazard Ratio (HR): 1.242, 95\% confidence interval (CI) 1.084-1.424, $p$ value $=0.002$ ]. Adding FFAs to the Cox model increased the C-statistic by $0.015(0.005-0.027)$. No significant difference in MACEs was observed between NGR and Pre-DM groups ( $p>0.05)$. When patients were categorized by both status of glucose metabolism and FFAs levels, medium and high FFAs were associated with significantly higher risk of MACEs in Pre-DM [1.736 (1.018-2.959) and 1.779 (1.012-3.126), all p-value < 0.05] and DM [2.017 (1.164-3.494) and 2.795 (1.619-4.824), all p-value<0.05].

Conclusions: The present data indicated that baseline FFAs levels were associated with the prognosis in DM and Pre-DM patients with CAD, suggesting that FFAs may be a valuable predictor in patients with impaired glucose metabolism.
\end{abstract}

Keywords: Free fatty acids, Pre-diabetes, Cardiovascular outcome

\section{Background}

Free fatty acids (FFAs)s, originated from adipose tissue and released by lipolysis of triglyceride, is the main source of energy in myocardium [1]. Elevated plasma FFAs often emerge in many metabolic diseases including obesity, type 2 diabetes (T2DM), hypertension and fatty liver disease [2-4]. In the meanwhile, FFAs could also induce endothelial dysfunction by increasing oxidative

\footnotetext{
*Correspondence: 13901010368@163.com

${ }^{\dagger}$ Jing-Lu Jin and Ye-Xuan Cao contributed equally to this work

Division of Dyslipidemia, State Key Laboratory of Cardiovascular Disease, Fu Wai Hospital, National Center for Cardiovascular Diseases, Chinese

Academy of Medical Sciences, Peking Union Medical College, BeiLiShi Road 167, Beijing 100037, China
}

stress, promoting inflammatory process and facilitating apoptosis of the endothelial cells [5]. Despite the surging evidences of the association between FFAs and cardiovascular outcomes in stable coronary disease (CAD) patients as well as healthy participants, current studies give no hint on the prognosis of FFAs in CAD patients with different glucose metabolism status [6, 7].

Prevalence of total diagnosed and undiagnosed diabetes in China had reached $10.9 \%$ and almost three times of patients were with pre-diabetes (Pre-DM) [8]. Patients with pre-DM also had high tendency to develop DM. According to Schrieks et al. data from the AleCardio (Effect of Aleglitazar on Cardiovascular Outcomes After Acute Coronary Syndrome in Patients With Type 2 Diabetes Mellitus) trial indicated that baseline FFAs

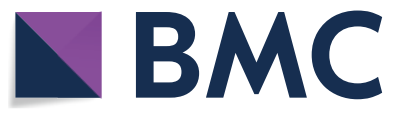

(c) The Author(s) 2019. This article is distributed under the terms of the Creative Commons Attribution 4.0 International License (http://creativecommons.org/licenses/by/4.0/), which permits unrestricted use, distribution, and reproduction in any medium, provided you give appropriate credit to the original author(s) and the source, provide a link to the Creative Commons license, and indicate if changes were made. The Creative Commons Public Domain Dedication waiver (http://creativecommons.org/ publicdomain/zero/1.0/) applies to the data made available in this article, unless otherwise stated. 
but not change in FFAs were associated with worse prognosis [9]. Moreover, pre-DM patients also have higher FFAs than those with normal glucose regulation (NGR) [10]. According to our previous studies, pre-DM did not increase cardiovascular risk alone but result in bad prognosis when combined with other metabolic disorder including hypertension and lipoprotein(a)-hyperlipoproteinemia $[11,12]$. In the current study, hence, we analyzed the joint effect of impaired glucose metabolism status and high FFAs on the outcomes of patients with stable CAD.

\section{Materials and methods}

\section{Study design and participants}

Our study complied with the Declaration of Helsinki and was approved by the hospital's ethical review board (Fu Wai Hospital and National Center for Cardiovascular Diseases, Beijing, China). Informed written consents were obtained from all patients enrolled in this study.

The details was described in the flowchart, from March 2011 to May 2015, 7535 patients from 20 provinces in China were admitted in Fuwai hospital and scheduled for coronary angiography because of angina-like chest pain and/or positive treadmill exercise test or clinically suspected CAD, Additional file 1: Figure S1. Among these patients, 312 were excluded for missing FFAs data and 569 were not angiography-proven CAD (coronary stenosis $\geq 50 \%$ of at least one coronary artery). Other patients were mainly excluded for following reasons: acute coronary syndrome (ACS), previous percutaneous coronary artery intervention (PCI) and bypass grafting $(\mathrm{CABG})$, heart failure, severe liver and/or renal insufficiency, thyroid dysfunction, systematic inflammatory disease and malignant disease. Patients were followed up every 6 month by means of interviewing directly or using telephone. Trained nurses or doctors who fulfilled the interview according to standard protocols. The major cardiovascular adverse events (MACEs) were cardiovascular mortality, non-fatal myocardial infarction (MI), stroke and post-discharge unplanned revascularization (PCI and CABG). Non-fatal myocardial infarction was diagnosed according to positive cardiac enzymes (troponins mainly) along with typical chest pain or electrocardiogram serial changes. Stroke was diagnosed according to precise medical records and imaging.

DM was diagnosed by fasting plasma glucose $(\mathrm{FPG}) \geq 7.0 \mathrm{mmol} / \mathrm{L}$ or the 2 -h plasma glucose of the oral glucose tolerance test $\geq 11.1 \mathrm{mmol} / \mathrm{L}$ or currently using oral antidiabetes drugs or insulin. Pre-DM was diagnosed when participants who did not meet the diagnostic criteria of DM but had a FPG ranges from 5.6 to $6.9 \mathrm{mmol} / \mathrm{L}, 2$-h glucose ranges from 7.8 to $11.0 \mathrm{mmol} / \mathrm{L}$, or haemoglobin A1c (HbA1c) level ranges from 5.7\% to $6.4 \%$. Patients who were without DM or Pre-DM were defined as normal glucose regulation (NGR) [13]. Hypertension was diagnosed for patients who provided previous medical history, who were currently taking antihypertensive drugs or who had systolic blood pressure $(\mathrm{SBP}) \geq 140 \mathrm{mmHg}$ and/or diastolic blood pressure $(\mathrm{DBP}) \geq 90 \mathrm{mmHg}$ for three or more consecutive times. Considering the skewed distribution pattern and narrow inter-tertile range of FFAs, we categorized level of FFAs with the cut off of 0.3 and $0.5 \mathrm{mmol} / \mathrm{L}$ according to previous study [9], Additional file 1: Figure S2. Information of other disease, family history, and prior therapy of every patient was collected from self-reported or hospital-recorded medical history.

\section{Laboratory analysis}

Blood samples were collected from each patient after fasting for at least 12-h. In consistent with our previous study, concentrations lipid parameters, including total cholesterol (TC), triglyceride (TG), low density lipoprotein cholesterol (LDL-C), high density lipoprotein cholesterol (HDL-C), and FFAs were measured using automatic biochemistry analyzer (Hitachi 7150, Tokyo, Japan) in an enzymatic assay. The concentrations of glucose were evaluated by enzymatic hexokinase method. HbA1c was measured using Tosoh Automated Glycohemoglobin Analyser (HLC-723G8, Tokyo, Japan).

\section{Evaluation of CAD severity}

As it was described previously, angiographic data were evaluated from catheter laboratory records by 3 experienced interventional cardiologists [11]. The Gensini score (GS) was calculated by standard means [14].

\section{Statistical analysis}

The values were expressed as the mean $\pm \mathrm{SD}$ or median (Q1-Q3 quartiles) for the continuous variables and the number (percentage) for the categorical variables. The Kolmogorov-Smirnov test was used to test the distribution pattern. $\mathrm{p}$ value for trend across glucose metabolism status in the continuous and categorical variables was examined by a generalized linear model and the Chi square test, respectively. The post hoc test between two groups were analyzed by Student t-test, Mann-Whitney U test (continuous variable) or Chi square test (categorical variable) where appropriate. The event-free survival rates among groups were estimated by the Kaplan-Meier method and compared by the log-rank test. Univariate and multivariate Cox regression analyses were performed to calculate the hazard ratios (HRs). In consistent with our previous study, the adjusted Cox models included previously reported risk factors as follows: age, sex, body 
mass index (BMI), smoking, hypertension, family history of coronary artery disease, Gensini score, left ventricular ejection fraction (LVEF), LDL-C, HDL-C, TG and baseline statins [11]. The efficiency of the models were assessed by $\mathrm{C}$-statistic. $\Delta \mathrm{C}$-statistic was used to interpret the incremental value of adding FFAs into original model. A p-value $<0.05$ was considered statistically significant. The statistical analyses were performed with SPSS version 21.0 software (SPSS Inc., Chicago, IL, USA) and R language version 3.5.2 (Feather Spray).

\section{Results}

Baseline characteristics

In the baseline characteristics, the age, BMI, glucose, HbA1c and TG were elevated according to the status of glucose metabolism from NGR to DM (all $p$ for trend $<0.001$, Table 1 ). The percentage of male patients was less in Pre-DM and DM groups while the proportion of patients with hypertension was higher among individuals with impaired glucose metabolism ( $\mathrm{p}$ for trend $<0.001$ ). Meanwhile, DM but not Pre-DM patients

Table 1 Baseline characters of participants according to glucose metabolism status

\begin{tabular}{|c|c|c|c|c|c|}
\hline Variables & $\begin{array}{l}\text { Total } \\
n=5433\end{array}$ & $\begin{array}{l}\text { NGR } \\
n=1039\end{array}$ & $\begin{array}{l}\text { Pre-DM } \\
n=2788\end{array}$ & $\begin{array}{l}\text { DM } \\
n=1606\end{array}$ & $\mathrm{p}$-value for trend \\
\hline \multicolumn{6}{|l|}{ Clinical characteristics } \\
\hline Age, years & $57.97 \pm 10.35$ & $54.45 \pm 10.54$ & $58.64 \pm 10.09$ & $59.09 \pm 10.19$ & $<0.001$ \\
\hline Male, & $3954(72.8)$ & $807(77.7)$ & $2009(72.2)$ & $11,139(70.9)$ & $<0.001$ \\
\hline $\mathrm{BMI}\left(\mathrm{kg} / \mathrm{m}^{2}\right)$ & $25.83 \pm 3.17$ & $25.31 \pm 2.99$ & $25.76 \pm 3.12$ & $26.30 \pm 3.14$ & $<0.001$ \\
\hline Overweight & $3291(60.5)$ & $581(55.9)$ & 1663 (59.6) & $1047(65.2)$ & $<0.001$ \\
\hline Obese & $505(9.3)$ & $72(6.9)$ & $249(8.9)$ & $184(11.5)$ & $<0.001$ \\
\hline Hypertension & $3451(63.5)$ & $598(57.6)$ & $1676(60.1)$ & $1177(73.3)$ & $<0.001$ \\
\hline Family history of CAD & 787 (14.5) & $172(16.6)$ & $379(13.6)$ & $236(14.7)$ & 0.066 \\
\hline Current Smoker & $2436(55.2)$ & $580(55.8)$ & $1559(52.0)$ & $858(53.4)$ & 0.248 \\
\hline Drinking & $1627(30.1)$ & $360(34.8)$ & $798(28.7)$ & $469(29.3)$ & 0.001 \\
\hline \multicolumn{6}{|l|}{ Laboratory findings } \\
\hline Glucose (mmol/L) & $5.71 \pm 1.69$ & $4.72 \pm 0.42$ & $5.21 \pm 0.73$ & $7.23 \pm 2.28$ & $<0.001$ \\
\hline $\mathrm{HbA1c}(\%)$ & $6.37 \pm 1.11$ & $5.39 \pm 0.24$ & $6.09 \pm 0.41$ & $7.51 \pm 1.32$ & $<0.001$ \\
\hline Creatinine ( $\mu \mathrm{mol})$ & $76.50 \pm 18.31$ & $76.40 \pm 15.52$ & $75.96 \pm 17.41$ & $77.50 \pm 21.24$ & 0.077 \\
\hline $\mathrm{TC}(\mathrm{mmol} / \mathrm{L})$ & $4.13 \pm 1.16$ & $4.01 \pm 1.13$ & $4.19 \pm 1.18$ & $4.11 \pm 1.15$ & 0.139 \\
\hline $\mathrm{HDL}-\mathrm{C}(\mathrm{mmol} / \mathrm{L})$ & $1.05 \pm 0.28$ & $1.05 \pm 0.29$ & $1.06 \pm 0.28$ & $1.02 \pm 0.27$ & 0.001 \\
\hline LDL-C (mmol/L) & $2.52 \pm 1.02$ & $2.45 \pm 1.06$ & $2.57 \pm 1.02$ & $2.48 \pm 1.00$ & 0.907 \\
\hline $\mathrm{TG}(\mathrm{mmol} / \mathrm{L})$ & $1.52(1.12-2.09)$ & $1.44(1.05-1.99)$ & $1.49(1.11-2.03)$ & $1.60(1.20-2.25)$ & $<0.001$ \\
\hline FFAs (mmol/L) & $0.40(0.30-0.53)$ & $0.40(0.30-0.48)$ & $0.40(0.29-0.49)$ & $0.46(0.35-0.57)$ & $<0.001$ \\
\hline LVEF (\%) & $63.37 \pm 8.36$ & $63.90 \pm 7.76$ & $63.24 \pm 8.54$ & $63.26 \pm 8.39$ & 0.093 \\
\hline Gensini score & $24(8-48)$ & $20(8-39)$ & $22(8-44)$ & $28(12-56)$ & $<0.001$ \\
\hline \multicolumn{6}{|l|}{ Medications } \\
\hline Baseline statins & 4294 (79.0) & 805 (77.5) & 2788 (79.9) & $1606(78.6)$ & 0.233 \\
\hline Follow-up statins & $5331(98.1)$ & $1011(97.3)$ & 2744 (98.4) & 1606 (98.1) & 0.077 \\
\hline Baseline aspirin & $2873(52.9)$ & $523(50.3)$ & 1488 (53.4) & $862(53.7)$ & 0.123 \\
\hline Follow-up aspirin & $5435(98.4)$ & $1039(98.1)$ & $2788(98.4)$ & 1606 (98.6) & 0.618 \\
\hline Baseline ACEIs/ARBs & $1540(28.3)$ & $295(28.4)$ & $778(27.9)$ & $467(29.1)$ & 0.623 \\
\hline Follow-up ACEIs/ARBs & $4661(85.8)$ & $886(85.3)$ & $2392(85.8)$ & $1383(86.1)$ & 0.552 \\
\hline Baseline $\beta$-blockers & $2873(52.9)$ & $523(50.3)$ & $1488(53.4)$ & $862(53.7)$ & 0.123 \\
\hline Follow-up $\beta$-Blockers & $4386(80.7)$ & $829(79.8)$ & $2253(80.8)$ & $1304(81.2)$ & 0.389 \\
\hline \multicolumn{6}{|c|}{ Baseline antidiabetes drugs } \\
\hline OADs & $937(17.2)$ & - & - & $937(58.3)$ & \\
\hline Insulin & $571(10.5)$ & - & - & $571(35.6)$ & \\
\hline
\end{tabular}

Data were expressed as mean $\pm S D$, median with 25 th and 75 th percentile or $n(\%)$

$\mathrm{p}$ for trend for the continuous and categorical variables was examined by a generalized linear model and the Chi square test

NGR normal glucose regulation, Pre-DM pre-diabetes mellitus, DM diabetes mellitus, BMI body mass index, HbA1c haemoglobin A1c, TC total cholesterol, TG triglyceride, $L D L-C$ low density lipoprotein cholesterol, $H D L-C$ high density lipoprotein cholesterol, FFAs free fatty acids, $L V E F$ left ventricular ejection fraction, $C A D$ coronary artery disease, $A C E I S$ ACE inhibitors, $A R B s$ angiotensin receptor blockers, OADs oral antidiabetes drugs 
had significantly lower levels of HDL-C than NGR population. There was no significant difference regarding smoking, family history of CAD, creatinine, LVEF, and proportion of statins and other medications (both baseline and follow-up) among the three groups ( $\mathrm{p}$ for trend $>0.05$ ).

\section{Predictive role of FFAs on MACCEs}

Over a median follow-up time of 6.7 years (4.0 to 8.2 years), 608 MACEs occurred (155 died, 71 suffered nonfatal MI, 209 had nonfatal strokes and 173 received unplanned revascularization). Univariate Cox proportional hazard regression analysis showed that $\log$ transformed FFA was associated with MACCEs [HR: 1.361, 95\% coincidence interval (CI) 1.191-1.554, p-value $<0.001$, Table 2]. In multivariate cox proportional hazard regression analysis (adjusted for age, sex, body mass index, hypertension, family history of CAD, smoke, HDL-C, LDL-C, log transformed TG, LVEF, GS and baseline statins) HR for the log transformed FFAs was 1.242 (95\% CI 1.084-1.424, p-value $=0.002)$. Both continuous and category FFAs were also associated with composite endpoints including cardiovascular mortality, nonfatal MI, and nonfatal stroke (adjusted medium FFAs: HR: 1.222, 95\% CI 0.929-1.607, p-value $>0.05$, adjusted high FFAs: HR: 1.727, 95\% CI 1.294-2.304, $\mathrm{p}<0.05$, adjusted $\log _{2}$ FFAs, HR: $1.240,95 \%$ CI $1.056-1.456, p<0.05$, Fig. 1a, b). The Cox prediction models of traditional risk factors for MACEs and composite endpoints were with
C-statistic values of 0.645 (95\% CI $0.618-0.672)$ and 0.626 (0.593-0.648) Addition of FFAs to original model showed significant improvement in C-statistic [ $\Delta \mathrm{C}$-statistic: 0.015 (0.005-0.027), $\mathrm{p}$-value $=0.019$ and 0.012 (0.003-0.020), $\mathrm{p}$-value $=0.028$, respectively, Table 3].

\section{Glucose metabolism, FFAs levels and cardiovascular outcomes}

The prevalence of MACEs in NGR, Pre-DM, and DM group was $8.6 \%, 10.2 \%$, and $14.6 \%$, respectively. Kaplan-Meier analysis showed that DM subjects had the lowest event-free survival rate among the 3 groups (p-value $<0.05$,) while there was no significant difference between that of Pre-DM and NGR groups ( $p$-value $>0.05$, Fig. 2a). By FFAs levels (low: FFAs $<0.3 \mathrm{mmol} / \mathrm{L}$, medium: $0.3 \leq$ FFAs $<0.5 \mathrm{mmol} / \mathrm{L}$, high: FFAs $\geq 0.5 \mathrm{mmol} / \mathrm{L}$ ), patients with high FFAs were least likely to be free of events (Fig. 2b). However, when the patients were evaluated according to both glucose metabolism and FFAs levels, Pre-DM plus medium FFAs, Pre-DM plus high FFAs, and DM plus medium FFAs and DM plus high FFAs groups had significantly lower cumulative event-free survival rates compared with the reference group (NGR plus FFAs group, Fig. $2 c$, all p-value $<0.05$ respectively).

Univariate Cox regression models showed that patients with DM had 1.677-fold higher risk of MACEs than NGR subjects [HR: 1.677, 95\% CI 1.312-2.143, $\mathrm{p}$-value $<0.05$, Table 4]. The significance of association did not change after adjustment for other variables. The

Table 2 Relation of the continuous plasma FFAs levels and cardiovascular risk factors with MACEs

\begin{tabular}{|c|c|c|c|c|}
\hline \multirow[t]{2}{*}{ Variables } & \multicolumn{2}{|c|}{ Univariate Cox regression } & \multicolumn{2}{|c|}{ Multivariate Cox regression } \\
\hline & HR $(95 \% \mathrm{Cl})$ & p-value & $\mathrm{HR}(95 \% \mathrm{Cl})$ & p-value \\
\hline Age & $1.024(1.016-1.032)$ & $<0.001$ & $1.020(1.010-1.029)$ & $<0.001$ \\
\hline Sex & $1.015(0.849-1.213)$ & 0.870 & - & \\
\hline BMI & $0.990(0.965-1.016)$ & 0.457 & - & \\
\hline LVEF & $0.975(0.962-0.987)$ & $<0.001$ & $0.979(0.971-0.988)$ & $<0.001$ \\
\hline Hypertension & $1.320(1.111-1.586)$ & 0.002 & $1.221(1.017-1.466)$ & 0.032 \\
\hline DM status & $1.352(1.201-1.522)$ & 0.020 & $1.200(1.060-1.359)$ & 0.004 \\
\hline Smoke & $1.005(0.857-1.179)$ & 0.951 & - & \\
\hline $\mathrm{FH}$ & $1.240(0.973-1.582)$ & 0.082 & & \\
\hline Log transformed TG & $1.038(1.980-1.100)$ & 0.205 & - & - \\
\hline $\mathrm{HDL}-\mathrm{C}$ & $1.142(0.856-1.522)$ & 0.376 & - & \\
\hline LDL-C & $1.014(0.937-1.097)$ & 0.730 & - & \\
\hline GS & $1.007(1.005-1.009)$ & $<0.001$ & $1.005(1.003-1.007)$ & $<0.001$ \\
\hline $\log _{2}$ FFAs & $1.361(1.191-1.554)$ & $<0.001$ & $1.242(1.084-1.424)$ & 0.002 \\
\hline Baseline Statin & $0.817(0.697-0.959)$ & 0.013 & $0.792(0.671-0.936)$ & 0.006 \\
\hline
\end{tabular}

Univariate and multivariate Cox proportional hazards regression analysis was performed to test statistical significance

MACEs were cardiovascular mortality, non-fatal myocardial infarction (MI), stroke and post-discharge unplanned revascularization

MACEs major cardiovascular adverse events, $B M I$ body mass index, $T G$ triglyceride, $L D L-C$ low density lipoprotein cholesterol, $H D L-C$ high density lipoprotein cholesterol, FFAs free fatty acids, LVEF left ventricular ejection fraction, DM diabetes mellitus, FH family history of coronary artery disease, GS gensini score 


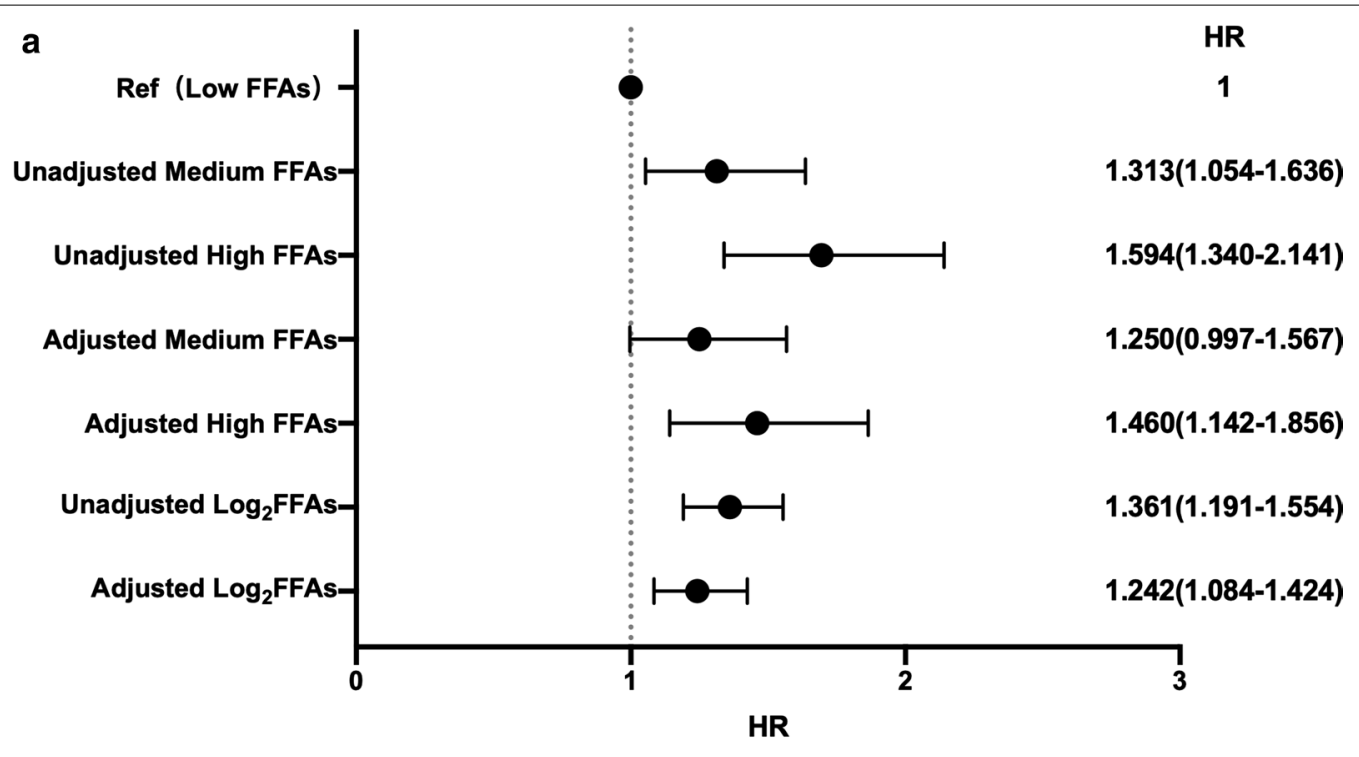

HR

b

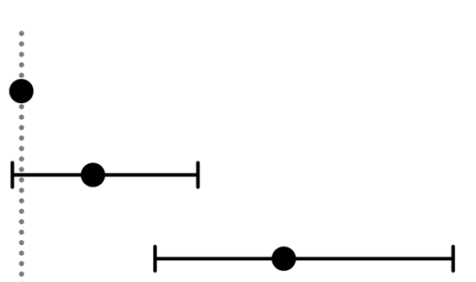

$1.261(0.967-1.644)$

Unadjusted Medium FFAs-

Ref (Low FFAs)

$$
-1
$$

Unadjusted High FFAs-
Adjusted Medium FFAs-

Adjusted Medium FFAs
Adjusted High FFAs

Unadjusted Log $\log _{2}$ FAs-

A

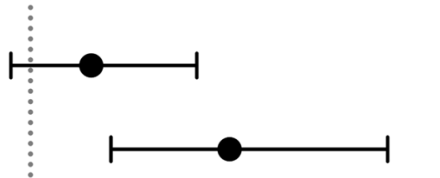

$1.958(1.488-2.576)$

$1.222(0.929-1.607)$

1.727(1.294-2.304)

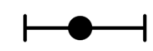

1.379(1.178-1.615)

$1.240(1.056-1.456)$

HR

Fig. 1 Prognostic value of continuous and category FFAs for a MACEs and $\mathbf{b}$ composite endpoints

Table 3 Incremental predictive values of FFAs for cardiovascular outcomes

\begin{tabular}{lll}
\hline & C-statistic (95\% Cl) & $\boldsymbol{\Delta C - s t a t i s t i c ~ ( 9 5 \% ~ C l ) ~}$ \\
\hline Original Model 1 & $0.645(0.618-0.672)$ & - \\
Original Model + $\log _{2}$ FFAs & $0.661(0.634-0.688)$ & $0.015(0.005-0.027)$ \\
Original Model 2 & $0.626(0.603-0.648)$ & - \\
Original Model 2 $+\log _{2}$ FFAs & $0.638(0.616-0.661)$ & $0.019(0.003-0.020)$ \\
\hline
\end{tabular}

C-statistic and $\Delta$ C-statistic were used to interpret efficiency of the models and the incremental value of adding FFAs into original model

Original model included age, sex, body mass index, smoking, hypertension, family history of coronary artery disease, Gensini score, left ventricular ejection fraction, low density lipoprotein cholesterol, high lipoprotein cholesterol, triglyceride and baseline statins. Model 1 indicates the C-statistic for MACEs (cardiovascular mortality, non-fatal myocardial infarction, stroke and post-discharge unplanned revascularization). Model 2 indicates the C-statistic for composite endpoints (cardiovascular mortality, non-fatal myocardial infarction, and stroke) 


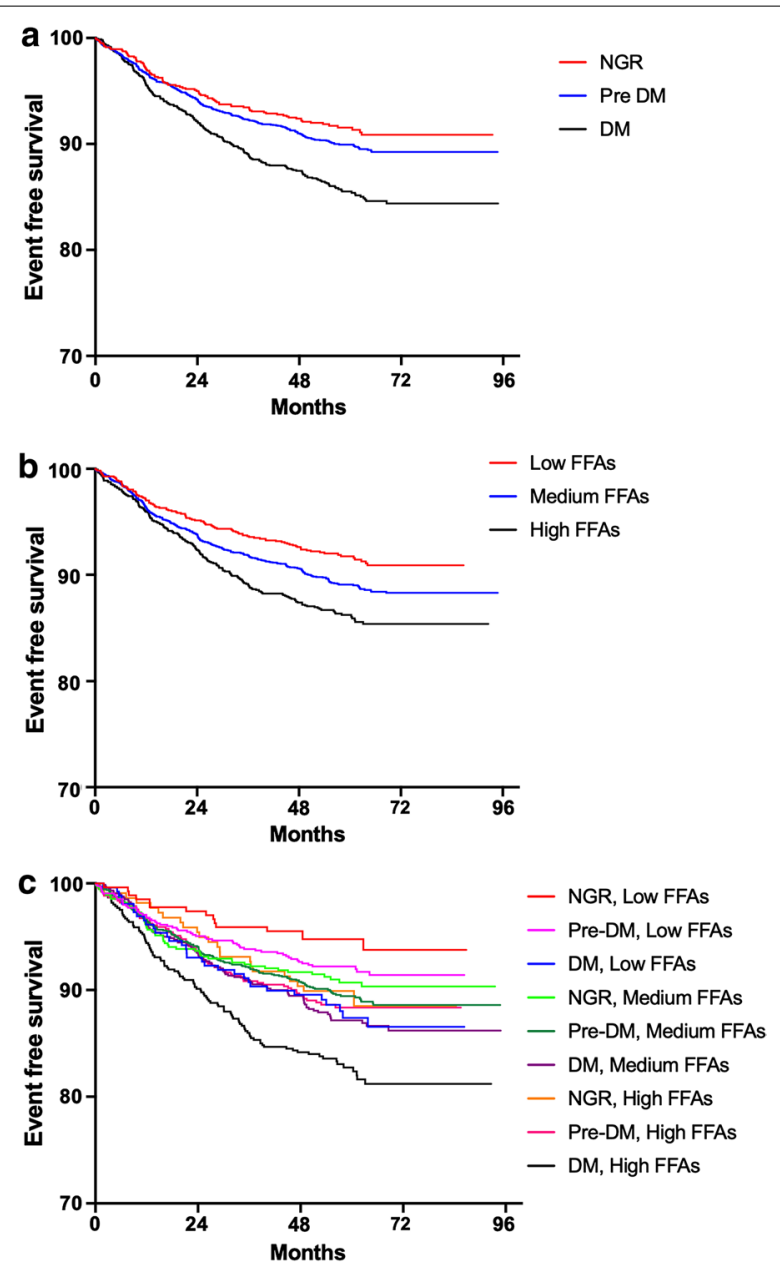

Fig. 2 Kaplan-Meier analysis according to a different glucose metabolism status; $\boldsymbol{b}$ different FFAs levels; $\mathbf{c}$ both status of FFAs levels and glucose metabolism

Table 4 Cardiovascular outcomes in different glucose metabolism status of the study participants

\begin{tabular}{lll}
\hline $\begin{array}{l}\text { Diabetic status or FFAs } \\
\text { level }\end{array}$ & HR $(\mathbf{9 5 \%} \mathrm{Cl})$ & \\
\cline { 2 - 3 } (n, events/subjects) & Unadjusted model & Adjusted model \\
\hline NGR (89/1039) & Ref & Ref \\
Pre-DM (285/2788) & $1.144(0.901-1.453)$ & $1.077(0.844-1.374)$ \\
DM $(234 / 1606)$ & $* 1.677(1.312-2.143)$ & $* 1.450(1.127-1.866)$
\end{tabular}

Univariate and multivariate Cox proportional hazards regression analysis was performed to test statistical significance

Model adjusted for age, sex, body mass index, smoking, hypertension, family history of coronary artery disease, Gensini score, left ventricular ejection fraction, low density lipoprotein cholesterol, high lipoprotein cholesterol, triglyceride, and baseline statin

$N G R$ normal glucose regulation, Pre-DM pre-diabetes mellitus, DM diabetes mellitus

* For $p$-value $<0.05$ presence of Pre-DM did not show increase in MACEs risk when compared with NGR group ( $\mathrm{p}$-value $>0.05$ ). When further stratified by both diabetic status and FFAs levels, medium and high FFAs were associated with significantly higher risk of MACEs in Pre-DM [1.736 (1.018-2.959) and 1.779 (1.012-3.126), all p-value <0.05] and DM [2.017 (1.164-3.494) and 2.795 (1.619-4.824), all p-value $<0.05$, Table 5].

\section{Discussion}

In this study, we investigated the impact of high FFAs on prognosis in stable, angiography-proven CAD patients with different glucose metabolism status. In consistent with our previous studies, FFAs was an independent predictor of MACEs. Adding FFAs in the traditional model also improved the predictive efficiency. Interestingly, Cox regression analysis showed that patients with DM but not those with Pre-DM had higher risk of MACEs when the

Table 5 FFAs levels in relation with cardiovascular events in patients with different glucose metabolism status

\begin{tabular}{|c|c|c|c|}
\hline \multirow[t]{2}{*}{ FFAs } & \multicolumn{3}{|c|}{$\mathrm{HR}(95 \% \mathrm{Cl})$} \\
\hline & $\begin{array}{l}\text { Events/ } \\
\text { subjects } \\
608 / 5433\end{array}$ & Crude Model & Adjusted Model \\
\hline \multicolumn{4}{|l|}{ NGR } \\
\hline Low FFAs & $15 / 268$ & Ref & Ref \\
\hline Medium FFAs & $51 / 553$ & $\begin{array}{l}1.665(0.936- \\
2.962)\end{array}$ & $1.543(0.865-2.752)$ \\
\hline High FFAs & $23 / 218$ & $\begin{array}{l}1.925(1.004- \\
3.689)^{*}\end{array}$ & $1.856(0.968-3.559)$ \\
\hline \multicolumn{4}{|l|}{ Pre-DM } \\
\hline Low FFAs & $61 / 747$ & $\begin{array}{l}1.457(0.828- \\
2.563)\end{array}$ & $1.271(0.721-2.242)$ \\
\hline Medium FFAs & $154 / 1430$ & $\begin{array}{l}1.936(1.140- \\
3.290)^{*}\end{array}$ & $\begin{array}{l}1.736(1.018- \\
2.959)^{*}\end{array}$ \\
\hline High FFAs & $70 / 611$ & $\begin{array}{l}2.098(1.201- \\
3.664)^{*}\end{array}$ & $\begin{array}{l}1.779(1.012- \\
3.126)^{*}\end{array}$ \\
\hline \multicolumn{4}{|l|}{$\mathrm{DM}$} \\
\hline Low FFAs & $32 / 259$ & $\begin{array}{l}2.245(1.216- \\
4.145)^{*}\end{array}$ & $\begin{array}{l}1.937(1.044- \\
3.594)^{*}\end{array}$ \\
\hline Medium FFAs & $96 / 740$ & $\begin{array}{l}2.354(1.366- \\
4.056)^{*}\end{array}$ & $\begin{array}{l}2.017(1.164- \\
3.494)^{*}\end{array}$ \\
\hline High FFAs & $106 / 607$ & $\begin{array}{l}3.308(1.926- \\
5.680)^{*}\end{array}$ & $\begin{array}{l}2.795(1.619- \\
4.824)^{*}\end{array}$ \\
\hline
\end{tabular}

Univariate and multivariate Cox proportional hazards regression analysis was performed in crude and adjusted models respectively

Model adjusted for age, sex, body mass index, smoking, hypertension, family history of coronary artery disease, Gensini score, left ventricular ejection fraction, low density lipoprotein cholesterol, high lipoprotein cholesterol, triglyceride, and baseline statin

Model adjusted for age, sex, body mass index, smoking, hypertension, family history of coronary artery disease, Gensini score, left ventricular ejection fraction, low density lipoprotein cholesterol, high lipoprotein cholesterol, triglyceride, and baseline statin

* For $p$-value $<0.05$ 
patients were categorized according to glucose metabolic status. When patients were divided into 9 groups according to both status of glucose metabolism and FFAs levels, patient with Pre-DM plus high FFAs and DM plus high FFAs had 1.779- and 2.795-fold increased risk of MACEs compared with that in subjects with NGR and low FFAs. Thus, our study firstly suggested the joint predictive value of DM status and FFAs levels on MACEs.

FFAs provide $\sim 70 \%$ of the energy required by myocardial metabolism. Increased plasma FFAs were harmful for cardiomyocytes because more oxygen was demanded in glycolysis [15]. FFAs could also impair the PI3 K pathway which blunts Akt activity and phosphorylation of endothelial nitric oxide synthase (eNOS) at Ser1177, resulting in vascular endothelial dysfunction [16, 17]. High level of plasma FFAs was one of the most common features in individuals with metabolic abnormality. In patients with diabetes or obesity, more FFAs were released from adipose tissue leading to downregulation of insulin responsive glucose transporter $4[16,17]$. In consistent with up-mentioned underlying mechanisms, level of FFAs was associated with presence and severity of hypertension. For example, in a cross-sectional study by Nagahama Study Group, the level of plasma FFAs was positively associated with brachial pulse pressure amplification and negatively related to augmentation index and central systolic $\mathrm{BP}^{2}$. In the Paris Prospective Study, the 90th percentile of fasting plasma FFAs concentration was associated with $58 \%$ higher risk of hypertension when compared with the 10th percentile group [18]. In the Cardiovascular Health Study, plasma high level of FFAs was associated with a higher incidence rate of heart failure in older adults [19]. Furthermore, levels of plasma FFAs could also independently predict the degree of stenosis in both carotid and coronary arteries [20, 21]. In primary prevention studies, the results about prognosis of FFAs in cardiovascular outcomes were inconsistent. In the Paris Prospective study FFAs concentrations were not related to cardiovascular death while elevated plasma FFAs were associated with both cardiovascular and noncardiovascular mortality in the Cardiovascular Health Study [7, 22]. More importantly, high FFAs can increase the ischemic damage to the myocardium when patients were with prior coronary stenosis [23]. Therefore, studies about the prognosis of FFAs in patients who manifested CAD might be in need.

Several studies also have demonstrated the predictive value of FFAs in patients with established CAD. Pliz et al. reported that FFAs were similarly associated with cardiac mortality in patients with angiographic-proven CAD, stable CAD and unstable CAD [6]. Similarly, Breitling and his colleague reported that very high FFAs might identify worse outcomes for patients with stable CAD [24]. It was demonstrated in AleCardio trial that a twofold higher baseline FFAs was directly associated with $17 \%$ higher risk of MACEs in patients with both T2DM and ACS [9]. The study by our group also reported that the HR for fourth FFAs quartile was 1.80 after adjustment for traditional cardiovascular risk factors when compared with that in the first FFAs quartile [25]. In the IMMEDIATE trial, very early intravenous glucose-insulin-potassium for ACS patients could suppress FFAs and ultimately result in less cardiac arrest and in-hospital mortality [26]. Our present study, aiming at providing more evidence on the therapeutic and predictive value of this crucial marker in stable CAD patients, investigated the association of plasma FFAs to MACEs in a larger population with longer follow-up years.

CAD was a common comorbidity and the leading cause of death in DM patients [27]. Combined evaluation of lipid markers may be helpful in the risk stratification of CAD patients with DM [28]. It was also reported by many studies that patients with Pre-DM and CAD also had higher risk of bad prognosis when they were combined with other metabolic disorders [11, 12]. Interestingly, high circulating FFAs may play a potential role in disturbing the glucose metabolism and causing Pre-DM or DM. Decreasing the beta oxidation of FFAs to promote a shift to glucose metabolism was one of the main benefit in metabolic modulation for DM patients [29]. According to Chen et al., in newly diagnosed T2DM, liraglutide administration could reduce plasma FFAs and suppress soluble vascular cell adhesion molecule-1 [30]. In the Multi-Ethnic Study of Atherosclerosis, serum levels of FFAs relate to the 10-year risk of T2DM and n-3 fatty acids might attenuate the risk [3]. In DM status, the impaired use of FFAs in myocardial metabolism causes intramyocardial lipid accumulation as well as many cardiac dysfunctions including impaired mitochondrial function, cardiac hypertrophy and contractile dysfunction [31]. Curiously, studying combination effect of high FFAs and DM or Pre-DM status might provide new insight into the cardiovascular and metabolic risk estimation. In the Nagahama Study concerning the relationship of FFAs and blood pressure, the combination of FFAs quartile and DM status increased the pulse pressure amplification to $4.9 \mathrm{mmHg}^{2}$. However, no study about the combined effect of high FFAs and glucose metabolic status on risk of MACEs was currently available. In the present study, we not only gave our concern on prognosis of FFAs in patients with stable CAD, but also paid our attention to the joint effect of high FFAs and Pre-DM or DM on cardiovascular outcomes. As the main novel findings of our study, patients with Pre-DM and high FFAs or DM and high FFAs had 1.779- and 2.795-fold higher risk of MACEs, respectively. Pre-DM plus medium FFAs 
but Pre-DM or medium FFAs alone had worse prognosis, indicating that higher level of FFAs was another metabolic disorder affecting the prognosis in Pre-DM patients.

The present study had several limitations. Firstly, this is a study among Chinese patients with stable CAD. Berkson bias and detection signal bias were inevitable in this secondary prevention cohort. More studies in patients with other ischemic heart disease might also provide new insights. Secondly, the FFAs measurement was only at the baseline. Although the plasma levels of FFAs were easily affected by medications, studies till now mostly reported positive results for baseline levels of FFAs. Thirdly, we did not assess the all metabolic factors and parameters about insulin resistance due to the features of patients in our study. Thereby, more study is needed to confirm our findings.

\section{Conclusions}

Our data indicated that plasma FFAs were independently associated with MACEs. More importantly, when patients were categorized into 9 groups according to FFAs levels and glucose metabolism status, coexistence of high FFAs multiplied the risk of worse outcome in patients with Pre-DM or DM. Our results might provide new information on the necessity of monitoring FFAs in Pre-DM and DM patients.

\section{Supplementary information}

Supplementary information accompanies this paper at https://doi. org/10.1186/s12933-019-0936-8.

Additional file 1: Additional Figure S1. Flowchart of the study. Figure S2. Distribution of FFAs levels.

\begin{abstract}
Abbreviations
T2DM: type 2 diabetes mellitus; Pre-DM: pre-diabetes; NGR: normal glucose regulation; $A D$ : atherogenic dyslipidemia; LDL-C: low density lipoprotein cholesterol; HDL-C: high density lipoprotein cholesterol; TG: triglyceride; TC: total cholesterol; FFAs: free fatty acid; CAD: coronary artery disease; MACEs: major adverse cardiovascular events; $\mathrm{PCl}$ : percutaneous coronary coronary artery intervention; CABG: coronary artery bypass grafting; HbA1c: haemoglobin A1c; GS: Gensini score; BMI: body mass index; LVEF: left ventricle ejection fraction.
\end{abstract}

\section{Acknowledgements}

The authors thank all the staff and participants of this study for their important contributions.

\section{Authors' contributions}

$J-L J$ and Y-XC completed the project, analyzed the data, and drafted the manuscript. J-JL designed the study, interpreted the data and contributed to critically revising the manuscript. N-QW, C-GZ and Y-LG contributed to recruitment of patients and clinical diagnosis of disease. YG, H-HL, R-XX, J-S and Q-D contributed to the procedure of laboratory examination. All authors have approved the final article. J-JL is the guarantor of this work and, as such, had full access to all the data in the study and takes responsibility for the integrity of the data and the accuracy of the data analysis. All authors read and approved the final manuscript.

\section{Funding}

This work was partially supported by the Capital Health Development Fund (201614035) and CAMS Major Collaborative Innovation Project (2016-I2M-1011) awarded to Dr. Jian-Jun Li, MD, Ph.D. The study sponsors did not participate in the study design; the collection, analysis, or interpretation of data; the writing of the report; or the decision to submit the paper for publication.

\section{Availability of data and materials}

The datasets used and/or analysed during the current study are not publicly available but are available from the corresponding author on reasonable request.

\section{Ethics approval and consent to participate}

Our study complied with the Declaration of Helsinki and was approved by the hospital's ethical review board (Fu Wai Hospital and National Center for Cardiovascular Diseases, Beijing, China). Informed written consents were obtained from all patients enrolled in this study.

\section{Consent for publication}

Not applicable.

\section{Competing interests}

The authors declare that they have no competing interests.

Received: 18 August 2019 Accepted: 23 September 2019

Published online: 14 October 2019

\section{References}

1. Gertz EW, Wisneski JA, Stanley WC, Neese RA. Myocardial substrate utilization during exercise in humans Dual carbon-labeled carbohydrate isotope experiments. J Clin Investig. 1988;82:2017-25.

2. Tabara Y, Takahashi Y, Kawaguchi T, Setoh K, Terao C, Yamada R, Kosugi S, Sekine A, Nakayama T, Matsuda F, Nagahama Study G. Association of serum-free fatty acid level with reduced reflection pressure wave magnitude and central blood pressure: the Nagahama study. Hypertension. 2014;64:1212-8.

3. Steffen BT, Steffen LM, Zhou X, Ouyang P, Weir NL, Tsai MY. n-3 fatty acids attenuate the risk of diabetes associated with elevated serum nonesterified fatty acids: the multi-ethnic study of atherosclerosis. Diabetes Care. 2015;38:575-80.

4. Lee MR, Park KI, Ma JY. Leonurus japonicus Houtt attenuates nonalcoholic fatty liver disease in free fatty acid-induced HepG2 cells and mice fed a high-fat diet. Nutrients. 2017;10:E20.

5. Authors/Task Force M, Ryden L, Grant PJ, Anker SD, Berne C, Cosentino F, Danchin N, Deaton C, Escaned J, Hammes HP, Huikuri H, Marre M, Marx N, Mellbin L, Ostergren J, Patrono C, Seferovic P, Uva MS, Taskinen MR, Tendera M, Tuomilehto J, Valensi P, Zamorano JL, Guidelines EP, Zamorano $J \mathrm{~L}$, Achenbach S, Baumgartner H, Bax JJ, Bueno H, Dean V, Deaton C, Erol C, Fagard R, Ferrari R, Hasdai D, Hoes AW, Kirchhof P, Knuuti J, Kolh P, Lancellotti P, Linhart A, Nihoyannopoulos P, Piepoli MF, Ponikowski P, Sirnes PA, Tamargo JL, Tendera M, Torbicki A, Wijns W, Windecker S, Document R, De Backer G, Sirnes PA, Ezquerra EA, Avogaro A, Badimon L, Baranova E, Baumgartner H, Betteridge J, Ceriello A, Fagard R, Funck-Brentano C, Gulba DC, Hasdai D, Hoes AW, Kjekshus JK, Knuuti J, Kolh P, Lev E, Mueller C, Neyses L, Nilsson PM, Perk J, Ponikowski P, Reiner Z, Sattar N, Schachinger V, Scheen A, Schirmer H, Stromberg A, Sudzhaeva S, Tamargo JL, Viigimaa M, Vlachopoulos C, Xuereb RG. ESC Guidelines on diabetes, pre-diabetes, and cardiovascular diseases developed in collaboration with the EASD: the Task Force on diabetes, pre-diabetes, and cardiovascular diseases of the European Society of Cardiology (ESC) and developed in collaboration with the European Association for the Study of Diabetes (EASD). Eur Heart J. 2013;34:3035-87.

6. Pilz S, Scharnagl H, Tiran B, Seelhorst U, Wellnitz B, Boehm BO, Schaefer JR, Marz W. Free fatty acids are independently associated with all-cause and cardiovascular mortality in subjects with coronary artery disease. J Clin Endocrinol Metab. 2006;91:2542-7.

7. Miedema MD, Maziarz M, Biggs ML, Zieman SJ, Kizer JR, Ix JH, Mozaffarian D, Tracy RP, Psaty BM, Siscovick DS, Mukamal KJ, Djousse L. Plasma-Free 
fatty acids, fatty acid-binding protein 4 , and mortality in older adults (from the Cardiovascular Health Study). Am J Cardiol. 2014;114:843-8.

8. Wang L, Gao P, Zhang M, Huang Z, Zhang D, Deng Q, Li Y, Zhao Z, Qin $X$, Jin D, Zhou M, Tang X, Hu Y, Wang L. Prevalence and ethnic pattern of diabetes and prediabetes in china in 2013. JAMA. 2017;317:2515-23.

9. Schrieks IC, Nozza A, Stahli BE, Buse JB, Henry RR, Malmberg K, Neal B, Nicholls SJ, Ryden L, Mellbin L, Svensson A, Wedel H, Weichert A, Lincoff AM, Tardif JC, Grobbee DE, Schwartz GG. Adiponectin, Free fatty acids, and cardiovascular outcomes in patients with type 2 diabetes and acute coronary syndrome. Diabetes Care. 2018;41:1792-800.

10. Toledo-Corral CM, Alderete TL, Richey J, Sequeira P, Goran MI, Weigensberg MJ. Fasting, post-OGTT challenge, and nocturnal free fatty acids in prediabetic versus normal glucose tolerant overweight and obese Latino adolescents. Acta Diabetol. 2015;52:277-84.

11. Liu HH, Cao YX, Li S, Guo YL, Zhu CG, Wu NQ, Gao Y, Dong QT, Zhao $X$, Zhang Y, Sun D, Li JJ. Impacts of prediabetes mellitus alone or plus hypertension on the coronary severity and cardiovascular outcomes. Hypertension. 2018;71:1039-46.

12. Jin JL, Cao YX, Zhang HW, Sun D, Hua Q, Li YF, Guo YL, Wu NQ, Zhu CG, Gao Y, Dong QT, Liu HH, Dong Q, Li JJ. Lipoprotein(a) and cardiovascular outcomes in coronary artery disease patients with prediabetes and diabetes. Diabetes Care. 2019;42:1312-8.

13. American Diabetes A. Diagnosis and classification of diabetes mellitus. Diabetes Care. 2011;34(Suppl 1):S62-9.

14. Gensini GG. A more meaningful scoring system for determining the severity of coronary heart disease. Am J Cardiol. 1983;51:606.

15. Stanley WC, Recchia FA, Lopaschuk GD. Myocardial substrate metabolism in the normal and failing heart. Physiol Rev. 2005;85:1093-129.

16. Saltiel AR, Kahn CR. Insulin signalling and the regulation of glucose and lipid metabolism. Nature. 2001;414:799-806.

17. Kim JA, Montagnani M, Koh KK, Quon MJ. Reciprocal relationships between insulin resistance and endothelial dysfunction: molecular and pathophysiological mechanisms. Circulation. 2006;113:1888-904.

18. Fagot-Campagna A, Balkau B, Simon D, Warnet JM, Claude JR, Ducimetiere $P$, Eschwege E. High free fatty acid concentration: an independent risk factor for hypertension in the Paris Prospective Study. Int J Epidemiol. 1998:27:808-13.

19. Djousse L, Benkeser D, Arnold A, Kizer JR, Zieman SJ, Lemaitre RN, Tracy RP, Gottdiener JS, Mozaffarian D, Siscovick DS, Mukamal KJ, Ix JH. Plasma free fatty acids and risk of heart failure: the Cardiovascular Health Study. Circ Heart Fail. 2013;6:964-9.

20. Taniguchi A, Nakai Y, Fukushima M, Teramura S, Hayashi R, Hama K, Marumoto K, Watanabe T, Yoshioka I, Sakaguchi K, Kishimoto H, Matsushita K, Okumura T, Tokuyama K, Nagasaka S, Sakai M. Ultrasonographically assessed carotid atherosclerosis in Japanese type 2 diabetic patients: role of nonesterified fatty acids. Metab, Clin Exp. 2002;51:539-43.
21. Yang RF, Zhang H, Wang Z, Liu XY, Lin Z. A study on the relationship between waist phenotype, hypertriglyceridemia, coronary artery lesions and serum free fatty acids in adult and elderly patients with coronary diseases. Immun Ageing. 2018;15:14.

22. Charles MA, Fontbonne A, Thibult N, Claude JR, Warnet JM, Rosselin G Ducimetiere P, Eschwege E. High plasma nonesterified fatty acids are predictive of cancer mortality but not of coronary heart disease mortality: results from the Paris Prospective Study. Am J Epidemiol. 2001;153:292-8.

23. Oliver MF, Opie LH. Effects of glucose and fatty acids on myocardial ischaemia and arrhythmias. Lancet (London, England). 1994;343:155-8.

24. Breitling LP, Rothenbacher D, Grandi NC, Marz W, Brenner H. Prognostic usefulness of free fatty acids in patients with stable coronary heart disease. Am J Cardiol. 2011;108:508-13.

25. Zhang HW, Zhao X, Guo YL, Zhu CG, Wu NQ, Sun J, Liu G, Dong Q, Li JJ. Free fatty acids and cardiovascular outcome: a Chinese cohort study on stable coronary artery disease. Nutr Metab (Lond). 2017;14:41.

26. Selker HP, Beshansky JR, Sheehan PR, Massaro JM, Griffith JL, D'Agostino RB, Ruthazer R, Atkins JM, Sayah AJ, Levy MK, Richards ME, Aufderheide TP, Braude DA, Pirrallo RG, Doyle DD, Frascone RJ, Kosiak DJ, Leaming JM, Van Gelder CM, Walter GP, Wayne MA, Woolard RH, Opie LH, Rackley CE, Apstein CS, Udelson JE. Out-of-hospital administration of intravenous glucose-insulin-potassium in patients with suspected acute coronary syndromes: the IMMEDIATE randomized controlled trial. JAMA. 2012;307:1925-33.

27. Naito R, Miyauchi K. Coronary artery disease and type 2 Diabetes Mellitus. Int Heart J. 2017;58:475-80.

28. Tani S, Yagi T, Atsumi W, Kawauchi K, Matsuo R, Hirayama A. Relation between low-density lipoprotein cholesterol/apolipoprotein B ratio and triglyceride-rich lipoproteins in patients with coronary artery disease and type 2 diabetes mellitus: a cross-sectional study. Cardiovasc Diabetol. 2017;16:123.

29. Opie LH. Metabolic management of acute myocardial infarction comes to the fore and extends beyond control of hyperglycemia. Circulation. 2008;117:2172-7.

30. Chen XM, Zhang WQ, Tian Y, Wang LF, Chen CC, Qiu CM. Liraglutide suppresses non-esterified free fatty acids and soluble vascular cell adhesion molecule-1 compared with metformin in patients with recent-onset type 2 diabetes. Cardiovasc Diabetol. 2018;17:53.

31. Ormazabal V, Nair S, Elfeky O, Aguayo C, Salomon C, Zuniga FA. Association between insulin resistance and the development of cardiovascular disease. Cardiovasc Diabetol. 2018;17:122.

\section{Publisher's Note}

Springer Nature remains neutral with regard to jurisdictional claims in published maps and institutional affiliations.
Ready to submit your research? Choose BMC and benefit from:

- fast, convenient online submission

- thorough peer review by experienced researchers in your field

- rapid publication on acceptance

- support for research data, including large and complex data types

- gold Open Access which fosters wider collaboration and increased citations

- maximum visibility for your research: over $100 \mathrm{M}$ website views per year

At BMC, research is always in progress.

Learn more biomedcentral.com/submissions 